\title{
Designing Physics Experiment and Assessment of Inquiry-Based Laboratory to Exercise Higher Order Thinking Skills
}

\author{
Setiya Utari ${ }^{1}$, Eka Cahya Prima ${ }^{2}$ \\ \{setiyautari@upi.edu ${ }^{1}$, ekacahyaprima@upi.edu ${ }^{2}$ \} \\ Department of Physics Education, Faculty of Mathematics and Science Education, Universitas \\ Pendidikan Indonesia, Bandung, Indonesia ${ }^{1,2}$
}

\begin{abstract}
This work aims to design the inquiry-based laboratory as well as the cookbook laboratory model to train student's high order thinking. The investigation train how scientists invented standard quantities of length, time, and mass through the inquiry activity approach. The model is a part of the experiment assessment models under the revised 2013 Indonesian curriculum. The model was successfully applied to K-10 in a Senior High School. The result shows that the best attainment of the model is very high with $(0.90)$ item validity as much as its reliability, good (0.50) discrimination power, and medium (0.62) difficulty index. Average students achieve $74 \%$ of the experiment process test including investigating problems, tools, method, procedure, data, analysis, and conclusion. We have found that students can elaborate their facts and theory to synthesize, generalize, explain, hypothesize, or reach some conclusion or interpretation through applying inquiry-based laboratory and training their high order thinking skills.
\end{abstract}

Keywords: Inquiry-based Laboratory, Cookbook based Laboratory, High order thinking

\section{Introduction}

There are several polemics regarding the optimization of the national examination. The unfairness assessment which uncovered all aspects of the educational and focused on the cognitive element makes a low-quality of the national examination. On the other hand, the government gave hints about arranging practical assessment. This assessment contains psychomotor and affective evaluation. However, most Indonesian teachers are not able to create a practical assessment, so that the preparation of practical evaluations is required to give new contributions for government in arranging of the national examination. Furthermore, Husain [1] denotes that the advancement of suitable teaching material that contains the inquiry process can help a teacher to keep students attention.

Trumper [2] asserts that "laboratory" is a common term for activities based on observations, tests, and experiments carried out by students. This activity will exercise four important aspects including (a) skills (using instruments, training inquiry skills, following instructions and communicating results), (b) concepts (opening representative ideas, applying theory to higher levels, and finding out new concepts), (c) the nature of science (understanding the nature of science, and knowing how scientists work), and (d) attitudes (accuracy, openness, curiosity, objectivity, reality, and cooperativeness). Wenning [3] also affirms that the laboratory experiment is necessary to construct a student's logic and to connect to his 
verification. Accordingly, designing of practical examination is essential, because it can evaluate some crucial aspects understood by students. Besides, He denotes that inquiry allows students to identify questions and develop and follow their procedures to answer those questions [4].

We investigate that most secondary students integrate their activity in physics laboratory through "recipe-type" instruction. Allie [5] denotes that its laboratory model followed by some instructions would be able to solve the main problem expressed at its beginning section. However, it will not affect to intensively generate an experiment report, so that we need to reformulate the laboratory task. Thus, its model can evaluate the student's experiment competences from finding the problem by expressing the conclusion in a guided inquiry activity setting. Consequently, laboratory activity has become an indivisible part of science education [6].

The physics chapter of physical quantity and unit is familiarly known by Indonesian secondary students. A teacher commonly conducts this part by describing the definition of units in the same way as the textbook description without telling how scientists discover those units. Therefore, this research has concernedly introduced the inquiry laboratory compared with the cookbook laboratory for secondary students to present how scientists find out a unit. At the end of the section, students communicate their results, and a teacher also clarifies the student's concept. An appealing experiment can increase students' attention as well as teachers' so that the teacher's creativeness is required to invent a useful experiment media [7]. Moreover, not only a teacher who provides a required teaching media but also students trained inventing the new unit standard themselves. Moreover, higher-order thinking requires students to employ and control the information and ideas in ways, so that student can convert their senses and implications, such as when students merge information and ideas to synthesize, generalize, explain, hypothesize, or reach some conclusion or interpretation [8]. Higher-order thinking supposes students to resolve problems and improve meaning themselves. In short, with an inquiry question provided by a teacher, student's high order thinking can be partially trained.

\section{Research Method}

This research was led to build an inquiry experiment model and "recipe-type" model. It will be served to introduce secondary students how scientist creates standards unit in physics. The quasi-experimental design with the control group was selected. The test was provided to students at K-10 senior high school. It would be constructed to examine student's inquiry activities such as how to define problems, how to select experiment tools, how to build a method, and how to write procedures for analyzing data and inscribing conclusions. This research will be compared with the cookbook method which contains three questions about data, analysis, and conclusion. Test results will be evaluated including validity, reliability, discriminating power, and difficulty index. The complete supplementary data regarding the instrument can be accessed through the link http://bit.ly/instrument-inquirylab. 


\section{Result and Discussion}

As Trumper [2] said that "laboratory" can apply the concept to the higher levels students used their last information regarding a kinematic concept and a density concept to investigate a problem provided by the teacher. The hierarchy of high order thinking proposed by Newman and Wehlege [9] was performed by students. The following sentences will describe the first experiment setting to discover the standard unit of length. Firstly, the student synthesizes all probabilities of free motion as well as compulsive motion to get the same result in a repetition process. It is an important criterion to invent a standard of measurement. We looked that students try to find its unit standard of length by doing several actions such as falling some spherical objects with different mass from a place with $1 \mathrm{~m}$ in height above a flat surface, comparing the motion of some objects with difference shapes to find the influence of its shape, and comparing type of object motion such as trajectory line and straight line to find its interval time when the objects touch a surface. All investigations have been recorded by a video recorder to get accurate data of interval time while the object is falling itself. Those explanations can be seen in Figure 1.

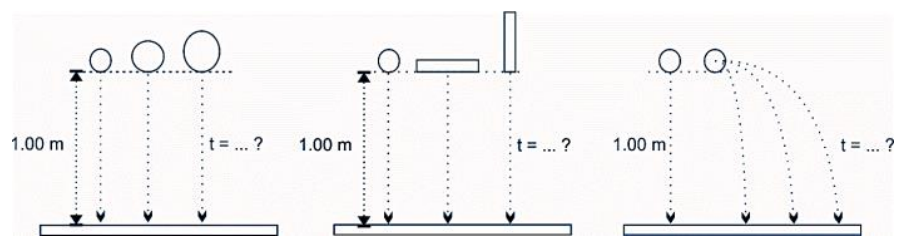

Fig. 1. Experiment setting to find the best choice in inventing the standard unit of length.

Secondly, students choose the shortest their interval time as the best selection because they consider that the obstacle factors can be neglected such as turbulence effect, medium viscosity, or object shape. In other words, the student can refer to a basic concept of free fall that there is a relationship among height, gravitational acceleration, and time, so that a student was trained to hypothesize its phenomenon. There is no data about the actual gravitational constant in our place. We consider that its constant is $9.8 \mathrm{~m} / \mathrm{s}^{2}$ so that students use this constant into their calculation. The time needed by free-fall objects onto the ground was also predicted by the student. Students also compared his experiment result $(0.49$ second is the shortest time required from $1 \mathrm{~m}$ of height the rest object into the ground) with a theoretical background $(0.45$ second is the time required from $1 \mathrm{~m}$ of altitude the rest object into the ground). The result is repeated to get a valid result. As a result, students have invented a simple standard unit of length themselves.

The following sentences will describe the following experiment setting to discover the standard unit of time. Firstly, like the first experiment, the student synthesizes all probabilities of free motion as well as compulsive motion to get the same result in a repetition process. It is a necessary principle to invent a standard of measurement. We observed that students attempt to discover its unit standard of time by falling some spherical objects with different mass from a place with $4.9 \mathrm{~m}$ in height above the ground. This study has been recorded by a video recorder to get accurate data of interval time while the object is falling itself. Students enthusiastically take some objects such as a tiny rock, a bottle, a book, and a sheet. Those explanations were depicted in Figure 2. 

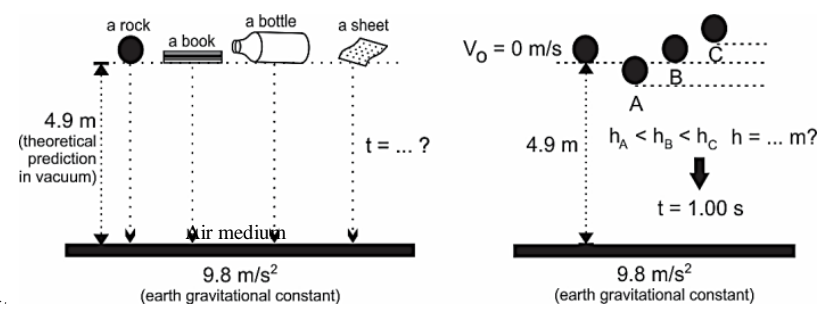

Fig. 2. Experiment setting to find the best choice in inventing the standard unit of time.

Secondly, students choose a tiny rock as the shortest of its interval time as and they consider that the air resistance can be neglected. Furthermore, the student mentions a physics concept about free fall and predicts the effect of air resistance in reducing the object acceleration, so that a student was trained to forecast its case. The student claims that its traveling rock might not be neglected at a long distance in the air. Although there is no data about the actual gravitational constant in our place, we still consider that its constant is close to $9.8 \mathrm{~m} / \mathrm{s}^{2}$. Therefore, students use this constant in their calculations. The distance needed by free-fall objects onto the ground was also predicted by the student. Most students predict "A" depicted in Figure 2 as the best answer. Students also compared his experiment result (4.7 meters is the farthest distance which results in $1.00 \mathrm{~s}$ traveling period of the elevated object into the ground) with a theoretically traveling in a vacuum (4.9 meters) if the gravitational constant is $9.8 \mathrm{~m} / \mathrm{s}^{2}$. As a result, students define a simple standard unit of time themselves and tell some weaknesses and suggestions of their invention.

The following sentences will describe the third experiment setting to discover the standard unit of mass. Firstly, students listened that the international standard unit of mass is the kilogram. The prototype was made from platinum-iridium compound due to anti-corrosion and 1 liter of pure water at $3.984{ }^{\circ} \mathrm{C}$. The teacher hunched that students will feel so hard to imagine this standardization criterion so that we need to rearrange the mass prototype standard at room temperature. Students supposedly determine the volume of water to achieve $1 \mathrm{~kg}$ of its mass. There are three questions whether its water volume at the room temperature is lower than at $4{ }^{\circ} \mathrm{C}$, or the water volume at the room temperature is the same as at $4{ }^{\circ} \mathrm{C}$, then water volume at the room temperature is higher than at $4{ }^{\circ} \mathrm{C}$. A student should previously know a concept about water anomaly and effect of thermal disturbance in changing the water volume, or a teacher should guide a student is experimenting with how the water density changes relative to changing its temperature. In the next section, students were trained to find other possibilities in alternative matters such as if we use a lot of rice to alter water as a mass prototype standard. Some predictions are described in the following sentences. Whether rice volume is larger than water volume, or its rice has the same amount as its water, or rice volume is smaller than water volume. Students ensure the rice's volume repeatability through 3 times making $1.00 \mathrm{~kg}$ of rice poured into the container. There is no data about a density of rice. Consequently, the student will compare their data with the density of water. In brief, students can find both densities proportionate to each volume. Those explanations were depicted in Figure 3.

Secondly, students concern about finding related concepts that support their investigations such as the effect of thermal disturbance to expand the volume, some important aspects in measurement, and kind of factors that make their research bias. Some students found that there is difficultness in ensuring 1 kilogram of water as well as rice because of the insensitiveness of the container relative to little change of water volume as well as rice's, and the student's 
carefulness to correctly read a scale. Based on their calculation of volume alteration affected by changing temperature and a data about the thermal expansion coefficient of water, they found that alteration volume theoretically calculated by student is $5.136 \times 10^{-3} \mathrm{~L}$. This value is relatively so hard to detect by scaled container as well as student so that students conclude that there is no significantly different volume of water between 2 conditions. Moreover, students investigate the volume of rice needed to fill the container. After 3 times of measurement, students conclude that the required rice volume as much as $1100 \mathrm{ml}$ to get $1 \mathrm{~kg}$ of rice. Students claim that it is a new standard to make $1 \mathrm{~kg}$ of mass. Furthermore, a teacher also checked the student's misconceptions about their result in the rice density. He said that if you calculate the density of rice, you will have a result that its density is lower than water density. But why the rice sinks in the water, whereas we have a previous concept that object will sink in the medium if its medium is lower density than the object. The question was illustrated in Figure 4.

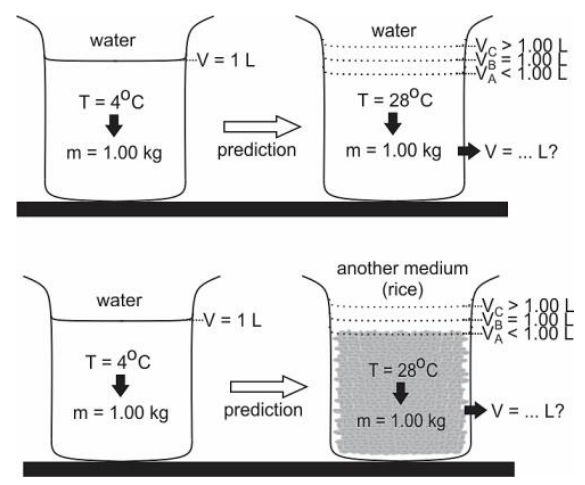

Fig. 3. Experiment setting to find the best choice in inventing the standard unit of mass.

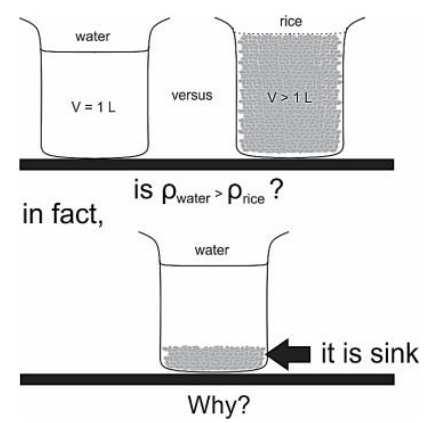

Fig. 4. Questions explored by a teacher to investigate student's high order thinking in the elaboration of a density concept and finding the standard unit of mass to clarify student's misconception.

Regarding its illustration, a teacher clarified that the phenomenon is caused by pore contamination in rice which filled the container. It should be reduced to minimize the bias effect in their result so that the rice powder is better than grain rice to invent the simple standard unit of mass. Moreover, a teacher still remembers his students that a standard should fulfill some criterions such as it has good resistance for the oxidation process, electrical shock, biological process, undamaged shape, and good repeatability. Therefore scientists always 
increase its criterion to make a high-quality prototype as a standard for calibrating a physical instrument. We look that students give their full attention in studying a standard unit in physics quantity as shown in Figure $\mathbf{5}$.

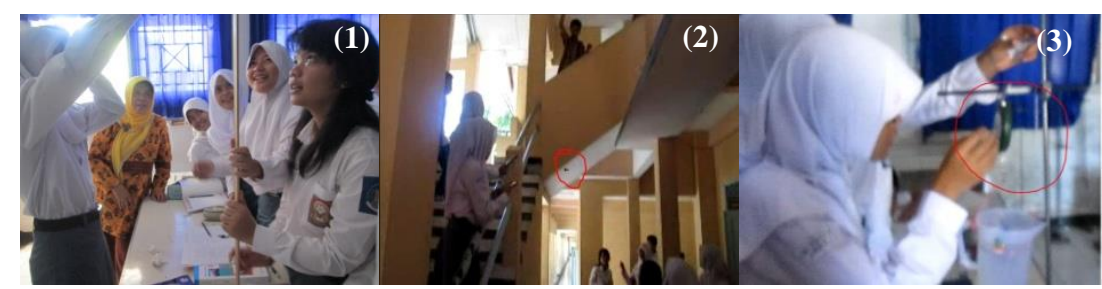

Fig. 5. Applying three experiment designs to invent the simple standard units of (1) length, (2) time, and (3) mass.

After implementing both experiment models, we evaluated the instrument and also found senior high school students profiles in inquiry activities as shown in Figure 6.

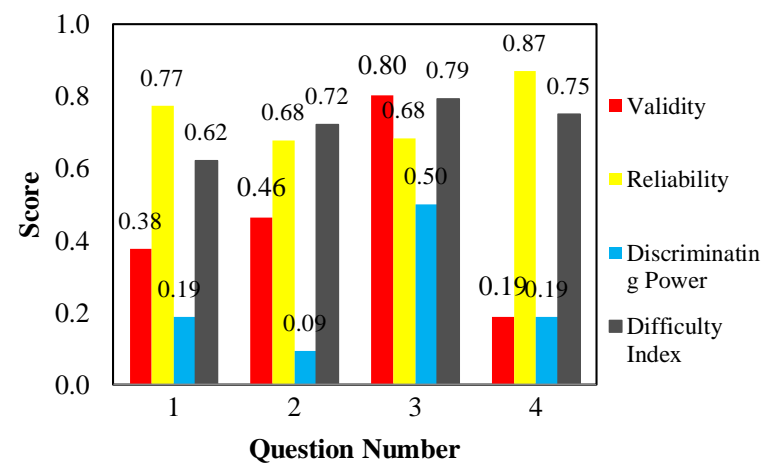

Fig. 6. Assessment analysis for the inquiry-based laboratory.

Assessment defines how the student can find the science problem in the unit chapter. The result indicates that item validity is low (0.38), item reliability is high (0.77), discriminating power is bad (0.19), and difficulty index is medium (0.62). We conclude that low validity and high reliability are certainly unsuitable to measure student competency in finding a problem. An alternative question might improve the assessment quality in finding a problem.

The second item which denotes how the student can select an experiment tool shows high item validity (0.46), high item reliability (0.68), bad discriminating power (0.09), and easy difficulty index (0.82). We conclude that its item is suitable to evaluate student competency in selecting the experiment tools and matters.

The third item defines how the student chooses a method to attack problems. The result denotes that item validity $(0.80)$ is as high as its reliability $(0.68)$, discriminating power is good (0.50), but difficulty index is easy (0.79). We conclude that this question is suitable to measure student competency in choosing the experimental method.

The fourth item defines how the student explains a procedure to clarify his experimental activity. The result indicates that item validity is very low $(0.19)$, item reliability is very high $(0.87)$, discriminating power is terrible (0.19), and difficulty index is easy $(0.75)$. We conclude that this question is suitable enough to appraise student competence in explaining the 
experimental procedures. But finding an alternative issue might improve the assessment quality in explaining experiment procedures.

According to our data and interpretations, we propose our physics experiment assessment through inquiry activity as a pure physics experiment model for senior high school students. Furthermore, some improvements in our model might make it better. Our experimental investigation is not only an inquiry-based laboratory but also a cookbook based laboratory. We assessed its instrument and also invented student characteristics at senior high school to get their preliminary reports, including data, analysis, and conclusion.

The complete interpretation data regarding our assessment was listed in Table 1. The first number in our recipe-type model defines how students can acquire the experimental data and ultimately report their activities on a working sheet. The result indicates that item validity is very high $(0.90)$, item reliability is high $(0.50)$, discriminating power is medium $(0.50)$, and the difficulty index is easy (0.74). We conclude that this question is suitable to analyze student competence in writing data. The second number of the test, which defines how the student can analyze the experiment data, has a medium item validity (0.60), high item reliability (0.64), a medium discriminating power (0.31), and easy difficulty index (0.75). We conclude that this question is sufficiently suitable to measure student competence to examine the experimental data. The third number question describes how students can arrange their experimental conclusion. The result indicates that its item validity is low $(0.22)$, item reliability is high (0.70), discriminating power is bad (0.06), and difficulty index is easy (0.84). We analyze this simplicity of this item because of hint availability in the beginning section. The activity is guided by the manual book as well as the teacher to facilitate student for simply attacking the problem. However, we conclude that this question is suitable to evaluate students' ability in concluding.

Table 1. Assessment data on inquiry-based laboratory and cookbook laboratory.

\begin{tabular}{|c|c|c|c|c|c|c|c|c|c|}
\hline \multirow{2}{*}{ Type* } & \multirow{2}{*}{ Number } & \multicolumn{3}{|c|}{ Validity } & \multicolumn{3}{|c|}{ Reliability } & \multicolumn{2}{|c|}{ Discriminating Power } \\
\hline & & Value & \multicolumn{2}{|c|}{ Interpretation } & Value & \multicolumn{2}{|c|}{ Interpretation } & Value & Interpretation \\
\hline \multirow[t]{4}{*}{ A } & 1 & 0.38 & \multicolumn{2}{|l|}{ Low } & 0.77 & \multicolumn{2}{|l|}{ High } & 0.19 & Bad \\
\hline & 2 & 0.46 & \multicolumn{2}{|l|}{ High } & 0.68 & \multicolumn{2}{|l|}{ High } & 0.09 & Bad \\
\hline & 3 & 0.80 & \multicolumn{2}{|l|}{ High } & 0.68 & \multicolumn{2}{|l|}{ High } & 0.50 & Good \\
\hline & 4 & 0.19 & \multicolumn{2}{|c|}{ Very low } & 0.87 & \multicolumn{2}{|c|}{ Very high } & 0.19 & Bad \\
\hline \multirow[t]{3}{*}{ B } & 1 & 0.90 & \multicolumn{2}{|c|}{ Very high } & 0.66 & \multicolumn{2}{|l|}{ High } & 0.50 & Medium \\
\hline & 2 & 0.60 & \multicolumn{2}{|c|}{ Medium } & 0.64 & \multicolumn{2}{|l|}{ High } & 0.31 & Medium \\
\hline & 3 & 0.22 & \multicolumn{2}{|l|}{ Low } & 0.70 & \multicolumn{2}{|l|}{ High } & 0.06 & Bad \\
\hline & & \multirow{2}{*}{ Type* } & \multirow{2}{*}{ Number } & \multicolumn{3}{|c|}{ Difficulty Index } & \multicolumn{2}{|c|}{ Mean Score } & \\
\hline & & & & Value & \multirow{2}{*}{\multicolumn{2}{|c|}{$\begin{array}{l}\text { Interpretation } \\
\text { Medium }\end{array}$}} & Score & Mark & \\
\hline & & \multirow[t]{4}{*}{ A } & 1 & 0.62 & & & 2.49 & 62 & \\
\hline & & & 2 & 0.72 & \multicolumn{2}{|l|}{ Easy } & 2.89 & 72 & \\
\hline & & & 3 & 0.79 & \multicolumn{2}{|l|}{ Easy } & 3.17 & 79 & \\
\hline & & & 4 & 0.75 & \multicolumn{2}{|l|}{ Easy } & 3.00 & 75 & \\
\hline & & \multirow[t]{3}{*}{ B } & 1 & 0.74 & Easy & & 2.97 & 74 & \\
\hline & & & 2 & 0.75 & Easy & & 3.00 & 75 & \\
\hline & & & 3 & 0.84 & Easy & & 3.34 & 84 & \\
\hline
\end{tabular}

*Type $A$ is the inquiry-based laboratory assessment and type B is the cookbook laboratory assessment 
In brief, we mention and give attention to an inquiry-based laboratory compared with the "recipe-type" model. Firstly, an inquiry-based laboratory model presents an appealing student's activities to develop their inquiry abilities started from how scientists construct a prototype for the standard unit through how scientist develop their models for a high-quality prototype. Secondly, students expand their higher-order thinking through elaborating the information and ideas in ways. Consequently, the student has converted their senses and implications, such as when students combine information and ideas to synthesize, generalize, explain, hypothesize, or reach some conclusion or interpretation. Indeed, all abilities have been trained through our experiment model.

\section{Conclusion}

We have concluded that the inquiry-based laboratory model could be implemented for a senior high school student to train high order thinking through inventing a simple standard of the unit in physics quantity. We found that students can elaborate their facts and theory to synthesize, generalize, explain, hypothesize, or reach some conclusion or interpretation through applying inquiry-based laboratory. Some competencies were built including recognizing scientist problems in inventing the unit standard, selecting some required tools to invent the new standard of the unit, deciding a suitable method, arranging experiment procedures, collecting data, analyzing data, and making a conclusion. We suggest developing the test continuously and using the same material at several schools to get more detailed characteristics.

\section{References}

[1] Hussain, A., Azeem, M. and Shakoor, A.: Physics teaching methods: scientific inquiry vs traditional lecture. International Journal of Humanities and Social Science, 1(19), pp. 269-276 (2011)

[2] Trumper, R.: The physics laboratory-a historical overview and future perspectives. Science \& Education, 12(7), pp. 645-670 (2003)

[3] Wenning, C. J.: Implementing inquiry-based instruction in the science classroom: A new model for solving the improvement-of-practice problem. Journal of Physics Teacher Education Online, 2(4), pp. 9-15 (2005)

[4] Wenning, C. J.: Experimental inquiry in introductory physics courses. Journal of Physics Teacher Education Online, 6(2), pp. 2-8 (2011)

[5] Allie, S., Buffler, A., Kaunda, L. and Inglis, M.: Writing-intensive physics laboratory reports: Tasks and assessment. The Physics Teacher, 35(7), pp. 399-405 (1997)

[6] Prima, E.C., Karim, S., Utari, S., Ramdani, R., Putri, E.R.R. and Darmawati, S.M.: Heat Transfer Lab Kit using Temperature Sensor based ArduinoTM for Educational Purpose. Procedia engineering, 170, pp. 536-540 (2017)

[7] Prima, E.C., Utari, S., Chandra, D.T., Hasanah, L. and Rusdiana, D.: Heat and temperature experiment designs to support students' conception on nature of science. JOTSE: Journal of technology and science education, 8(4), pp. 453-472 (2018)

[8] Mahoney, J.W. and Harris-Reeves, B.: The effects of collaborative testing on higher order thinking: Do the bright get brighter? Active Learning in Higher Education, 20(1), pp. 25-37 (2019)

[9] Newmann, F.N. and Wehlege, G.G.: Five Standards of Authentic Instruction. Association For Supervision and Curriculum Development, 50(7), pp. 8-12 (1993) 